\title{
Prevalencia y variables asociadas a la transmisión de Enterobius vermicularis en niños en edad preescolar y escolar en dos municipios de Cundinamarca, Colombia
}

\author{
Julio Cesar Giraldo Forero ${ }^{a}$ Lina María Pinzón Triana ${ }^{b}$ \\ - Laura Rodríguez Fonsecab - Valentina Vega Díaz
}

\begin{abstract}
Resumen: El presente artículo busca determinar la prevalencia de Enterobius vermicularis y variables asociadas en población preescolar y escolar del área urbana del municipio de Chaguaní y dos jardines en Cajicá, Cundinamarca, Colombia. Para esto se realizó un estudio descriptivo, de corte transversal con toma única de muestra en la población mencionada en 44 y 42 participantes, respectivamente, cuyos padres aceptaron participar voluntariamente firmando un consentimiento para desarrollar una encuesta epidemiológica estructurada, que indagó condiciones socioeconómicas, epidemiológicas y medioambientales. El diagnóstico parasitológico se realizó con la técnica de Graham. Los participantes se distribuyeron por grupo etario: el primero estuvo conformado por 76 niños $(88,4 \%)$ con edades entre 1,5 y 8 años; el segundo, por 7 niños mayores de 8 años (8,1\%), y el tercero, por 3 niños $(3,5 \%)$ sin información al respecto. La variable con significancia estadística $(p \leq 0.05)$ y posible asociación protectora fue la asistencia médica trimestral y como posible riesgo el contacto con fómites. Así, se encontró que la oxiuriasis es una parasitosis prevalente en la población preescolar y escolar, asociada a condiciones higiénico-sanitarias deficientes.
\end{abstract}

Palabras clave: Enterobius vermicularis; técnica de Graham; oxiuriasis, Colombia

Fecha de recepción: 30 de agosto de 2018 Fecha de aprobación: 10 de diciembre de 2018

Cómo citar: Giraldo Forero JC, Rodríguez Fonseca LC, Pinzón Triana LM, Vega Díaz V. Prevalencia y variables asociadas a la transmisión de Enterobius vermicularis en niños en edad preescolar y escolar en dos municipios de Cundinamarca, Colombia. Revista Med. 27(1): 17-27. Disponible en: https://revistas. unimilitar. edu.co/index.php/rmed/article/view/3916

a Líder del Grupo de Investigación en Parasitología y Microbiología Tropical - GIPAMT. Docente investigador programa profesional Biología. Universidad INCCA de Colombia. Docente Ciencias Básicas Universidad Militar Nueva Granada - Facultad de Medicina. Correo electrónico: julio.giraldo@unimilitar.edu.co

b Médico Universidad Militar Nueva Granada. 


\title{
Prevalence and Variables Associated with Enterobius vermicularis Transmission in Preschool and School Children in Two Municipalities of Cundinamarca, Colombia
}

\begin{abstract}
This article seeks to determine the prevalence of Enterobius vermicularis and associated variables in preschool and school population in the urban area of the municipality of Chaguani and two kindergartens in Cajicá, Cundinamarca, Colombia. To this end, a descriptive, cross-sectional study was conducted by taking a single sample from 44 and 42 participants from such populations, respectively, whose parents agreed to participate voluntarily by signing a consent to respond to a structured epidemiological survey. This survey inquired into their socioeconomic, epidemiological, and environmental conditions. Parasitological diagnosis was made using Graham's test. Participants were distributed by age group: the first one was made up of 76 children ( $88.4 \%$ ) between 1.5 and 8 years old; the second one, of seven children ( $8.1 \%$ ) over 8 ; and the third one, of three children (3.5\%) with no age information. A statistically significant variable $(p \leq 0.05)$ and possible protection factor was quarterly medical care. A possible risk factor was contact with fomites. Therefore, it was found that oxyuriasis is a prevalent form of parasitosis in preschool and school population associated with poor hygienic and sanitary conditions.
\end{abstract}

Keywords: Enterobius vermicularis; Graham's test; oxyuriasis, Colombia

\section{Prevalência e variáveis associadas à transmissão de Enterobius vermicularis em crianças pré-escolares e escolares em dois municípios de Cundinamarca, Colômbia}

Resumo: Este artigo pretende determinar a prevalência de Enterobius vermicularis e de variáveis associadas em população pré-escolar e escolar da área urbana do município de Chaguaní e de dois jardins de infância em Cajicá, Cundinamarca, Colômbia. Para isso, foi realizado um estudo descritivo, de corte transversal, com tomada única de amostras na população em 44 e 42 participantes, respectivamente, cujos responsáveis aceitaram a participar de forma voluntária e assinaram o consentimento para responder a um questionário epidemiológico estruturado, que indagou sobre condições socioeconômicas, epidemiológicas e meio ambientais. O diagnóstico parasitológico foi realizado com a técnica Graham. Os participantes foram distribuídos por grupo de idade: o primeiro formado por 76 crianças (88,4\%) entre 1,5 e 8 anos; o segundo, por 7 crianças com maios de 8 anos $(8,1 \%)$, e o terceiro, por 3 crianças $(3,5 \%)$ sem informação a respeito. A variável com significância estatística $(p \leq 0,05)$ e possível associação protetora foi a assistência médica trimestral e, como possível risco, o contato com fômites. Assim, foi verificado que a oxiuriase é uma parasitose prevalente na população pré-escolar e escolar, associada a condições higiênico-sanitárias deficientes.

Palavras-chave: Enterobius vermicularis; técnica de Graham; oxiuriase, Colômbia 


\section{Introducción}

La oxiuriasis, infección parasitaria causada por Enterobius vermicularis, está asociada a factores predisponentes como bajas condiciones socioeconómicas y costumbres poco higiénicas, que son frecuentes en países en desarrollo $(1,2)$. Las áreas rurales y urbanas marginales son susceptibles de adquirir esta infección, siendo la principal vía de transmisión de persona a persona, por migración de las hembras para ovopositar y adherir los huevos en la región perianal. Esto ocasiona prurito y su acumulación debajo de las uñas del hospedero, lo que lleva a reinfectarse o a infectar a otras personas de manera directa o por contaminación de objetos (fómites) $(3,4)$.

En algunos casos, la infección suele ser asintomática; sin embargo, algunos niños pueden presentar síntomas como prurito anal, sensación de cuerpo extraño, irrupción del patrón de sueño y bruxismo, lo que ocasiona alteraciones en la conducta y deteriora el desarrollo social y académico $(5,6)$. Existen reportes de migración e invasión del parásito a la vía genitourinaria y ovarios en la población femenina: peritoneo, pared intestinal, apéndice cecal, tracto biliar, hígado, pulmón y ocular, lo cual genera complicaciones severas ( 7 , $8,9,10)$; también se cree que la enterobiasis puede estar asociada a parasitosis como Ascaris lumbricoides, Strongyloides stercoralis y en algunos casos en coinfección con Dientamoeba fragilis, por el hallazgo de ADN del protozoo en la superficie de los huevos de E. vermicularis (11).

En Colombia las prevalencias para oxiuriasis en la población infantil, con valoración por el método de Graham, oscilan entre el 2,5\% y el 32,9\% $(1,12,13)$, con una prevalencia general en coproparasitología del 1\%, según la Encuesta Nacional de Parasitismo Intestinal en Población Escolar Colombia 2012-2014 (ENPI) (14, 15). Datos epidemiológicos reportados en estudios realizados en países como Argentina, Venezuela, Perú, Ecuador, México, Cuba, Korea, Turquía y Taiwan, entre otros, denotan lo prevalente que es la parasitosis a nivel mundial, con tasas que oscilan entre $0,62 \%$ al $69,9 \%(16,17,18,19)$.
El objetivo de esta investigación fue determinar la prevalencia de E. vermicularis e identificar posibles variables asociadas a la transmisión, la población preescolar y escolar de los municipios de Chaguaní y Cajicá, Cundinamarca, Colombia en el primer semestre de 2016.

\section{Materiales y métodos}

Para esta investigación se trazó la metodología que permitiera la recolección de información ecoepidemiológica y toma de muestra, con la técnica de referencia establecida por la oms.

\section{Área y población de estudio}

Se realizó un estudio descriptivo, de corte transversal, de febrero a junio de 2016, en población preescolar y escolar de la zona urbana de Chaguaní, municipio localizado en el occidente del departamento de Cundinamarca, con una extensión de $142 \mathrm{~km}^{2}$, y siendo el $0,139 \mathrm{~km}^{2}$ de área urbana. La población hace parte de la provincia del Magdalena centro, su cabecera se localiza a los $4^{\circ} 57^{\prime}$ de longitud Norte y $74^{\circ} 36^{\prime}$ de longitud Oeste; a 1.050 m.s.n.m., y cuenta con temperatura promedio de $24^{\circ} \mathrm{C}$ (20). El plan de desarrollo municipal (PDM 2012-2015) reportó para el área urbana una población de 731 habitantes, de los cuales 150 son niños con edades de 0 a 14 años. Al servicio de estos hay un centro de salud nivel 1, razón por la cual las historias clínicas se encuentran registradas en el hospital del municipio vecino, Guaduas, lo que lleva al desconocimiento de la prevalencia parasitaria. En el estudio participó toda la población infantil preescolar y escolar, con 52,3\% (23/44) del género masculino y $47,7 \%$ (21/44) femenino, con edades entre 1,5 y 10 años (21).

En Cajicá participaron 42 menores de edad preescolar, 57,1\%(24/42) del género masculino y $42,9 \%(18 / 42)$ del femenino, con edades entre 3 y 5 años. Los menores asisten a los jardines infantiles Sandra Ceballos del Instituto Colombiano de Bienestar Familiar (ICBF) y la Fundación Santa Coloma de Cajicá. El municipio se encuentra ubicado en la Sabana centro, a $04^{\circ}$ 55' latitud Norte y $74^{\circ} 01^{\prime}$ longitud Oeste, y tiene 53.397 habitantes. 
El área urbana está situada a $2558 \mathrm{~m} \mathrm{s.} \mathrm{n.} \mathrm{m.,} \mathrm{y} \mathrm{pre-}$ senta una temperatura promedio de $14^{\circ} \mathrm{C}$. Para la atención en salud cuenta con tres centros hospitalarios. Los municipios de Chaguaní y Cajicá están localizados en la provincia biogeográfica norandina colombiana $(13,14)$.

Para esta investigación, los criterios de inclusión fueron: población infantil de 1 a 10 años de edad, asentimiento voluntario de los menores, consentimiento informado de los padres y toma de la muestra por personal médico especializado empleando la técnica de Graham; además, la población seleccionada no tenía que haber sido desparasitado en el último mes; de esta manera, se dio cumplimiento a lo estipulado en la Ley 8/2015 de protección al menor (22).

\section{Recolección de datos}

Con el empleo de una ficha epidemiológica estructurada, se encuestaron a los padres de familia o representantes del menor, a quienes se les instruyó con charlas educativas sobre parasitismo intestinal e implicación en la salud de la población infantil, socialización y firma voluntaria del consentimiento informado, y de esta manera hacerlos partícipes del estudio; además, se contó con el asentimiento de los niños. Se indagó acerca de condiciones socioeconómicas, posesión de electrodomésticos, acceso a servicios de salud y tratamientos médicos; asimismo, sobre condiciones epidemiológicas y medioambientales (presencia de vectores mecánicos en el entorno o en el lugar de residencia, poseer sistemas de acueducto y alcantarillado en las viviendas), practicas higiénico-sanitarias familiares como lavado de manos frecuente, lavado de hortalizas y verduras para el consumo, hábitos de juego que involucren contacto con tierra, onicofagia, presencia de mascotas en el lugar de residencia, eliminación de excretas y caminar sin calzado.

\section{Diagnóstico parasitológico}

Se empleó la técnica de Graham tomando una sola muestra, y para la lectura se examinaron las muestras microscópicamente con observación por triple ciego, objetivos de 10x y 40x, utilizando el condensador con baja intensidad lumínica para conseguir un mejor contraste. El hallazgo de huevos característicos del agente etiológico en el frotis colectado fue indicador de positividad para Enterobius vermicularis (ver figura 1) (23). Los padres de familia o representantes del menor fueron informados de los resultados y en los casos positivos se les suministró tratamiento.

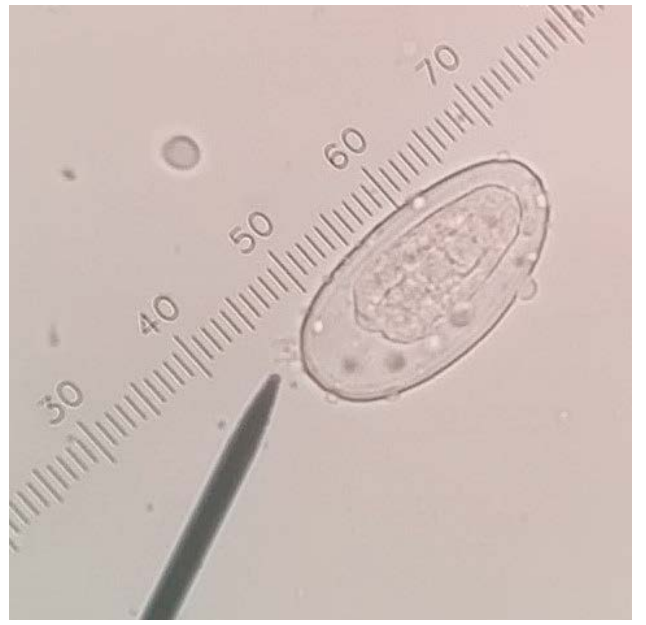

Figura 1. Huevo E. vermicularis $40 x$ con solución de lugol Fuente: los autores.

\section{Análisis estadístico}

Los datos del estudio fueron tabulados en tablas Excel-Windows y analizados mediante el empleo del programa Epi-Info, v.7.2.2.6. Se calcularon los valores de ji cuadrado, frecuencias y OR, lo que permitió establecer la posible relación de asociación entre las variables analizadas y la presencia de E. vermicularis en la población estudiada (24).

\section{Resultados}

Los 86 participantes se distribuyeron por grupo etario: el primero estuvo conformado por 76 niños $(88,4 \%)$ con edades entre 1,5 y 8 años, 7 mayores de 8 años $(8,1 \%)$ y $3(3,5 \%)$ sin información al respecto.

El 22,1\% (19/86) de los niños fue diagnosticado positivo, con el 27,3\% (12/44) correspondiente a Chaguaní y el 16,7\% (7/42) a Cajicá. Por género el $63,1 \%(12 / 19)$ correspondió al femenino y $21 \%$ (4/19) al masculino; del 15,8\% restante (3/19), por omisión de datos en la encuesta, se desconoce el género. Por edades, 94,7\% (18/19) corresponde a niños 
entre 1,5 y 8 años) y 5,3\% (1/19) a niños mayores de 8 años. En la tabla 1 se presenta el perfil sociodemográfico y otras variables evaluadas en el estudio.

Se identificaron las variables con significancia estadística y posible factor de riesgo para la adquisición de la parasitosis, como poseer computador en la vivienda del menor, lo que haría suponer que este elemento adquiere un papel de fómite $(\mathrm{p}=0,025, \mathrm{OR}=7,5)$ y condición de hacinamiento en el dormitorio $(\mathrm{p}=0,04, \mathrm{OR}=2,8)$. Las variables con significancia estadística y factor de carácter protector para no adquirir la parasitosis fueron los controles médicos mensuales $(\mathrm{p}=0,001, \mathrm{OR}=3,6) \mathrm{y}$ trimestrales $(\mathrm{p}=0,05, \mathrm{OR}=2,8)$, como también estar afiliado al Sistema de Selección Beneficiario para Programas Sociales (Sisbén) ( $\mathrm{p}=0,027, \mathrm{OR}=4,0$ ).

Tabla 1. Perfil sociodemográfico de la población preescolar y escolar del área urbana de los municipios de Chaguaní y grupo piloto de Cajicá

\begin{tabular}{|c|c|c|c|}
\hline $\begin{array}{l}\text { Perfil sociodemográfico } \\
\text { IC } 95 \%\end{array}$ & $n(86)$ & $\%$ & OR \\
\hline \multicolumn{4}{|l|}{ Género } \\
\hline Masculino & 47 & 54,7 & - \\
\hline Femenino & 39 & 45,3 & - \\
\hline \multicolumn{4}{|l|}{ Edad años } \\
\hline 1,5 a 8 & 76 & 88,4 & - \\
\hline$>8$ & 7 & 8,1 & - \\
\hline Indeterminado & 3 & 3,5 & - \\
\hline \multicolumn{4}{|l|}{ Estratificación social } \\
\hline Televisor & 61 & 71,0 & - \\
\hline Nevera & 63 & 73,3 & - \\
\hline Equipo de sonido & 39 & 45,3 & 0,3 \\
\hline Computador & 20 & 23,3 & $7,5^{*}$ \\
\hline Lavadora & 53 & 61,6 & 0,6 \\
\hline Ninguno & 23 & 26,7 & - \\
\hline \multicolumn{4}{|l|}{ Servicio de Salud } \\
\hline Prepagada & 8 & 9,3 & 0,7 \\
\hline Subsidiada & 31 & 36,0 & 0,3 \\
\hline Sisbén & 23 & 26,7 & $4,0^{*}$ \\
\hline Ninguno & 24 & 28,0 & - \\
\hline \multicolumn{4}{|l|}{ Control médico } \\
\hline Mensual & 18 & 21,0 & $3,6^{*}$ \\
\hline Trimestral & 20 & 23,3 & $2,8^{*}$ \\
\hline Semestral & 10 & 11,6 & 0,4 \\
\hline Anual & 10 & 11,6 & 0,8 \\
\hline Sin control & 28 & 32,6 & - \\
\hline \multicolumn{4}{|l|}{ Suministro de antiparasitarios } \\
\hline Domésticos & 2 & 2,3 & 0,6 \\
\hline Adquirido en dispensario & 20 & 23,3 & 0,7 \\
\hline Formulado & 37 & 43,0 & 0,4 \\
\hline Ninguno & 27 & 21,4 & - \\
\hline
\end{tabular}




\begin{tabular}{|c|c|c|c|}
\hline $\begin{array}{l}\text { Perfil sociodemográfico } \\
\text { IC } 95 \%\end{array}$ & $\mathrm{n}(86)$ & $\%$ & OR \\
\hline \multicolumn{4}{|l|}{ Suministro de agua } \\
\hline Acueducto & 61 & 71,0 & 0,3 \\
\hline Aljibe & 20 & 23,3 & - \\
\hline Pozo de reserva & 4 & 4,7 & - \\
\hline Ninguno & 1 & 1,2 & - \\
\hline \multicolumn{4}{|l|}{ Agua de consumo } \\
\hline Tratada acueducto & 53 & 61,6 & - \\
\hline Hervida & 49 & 57,0 & - \\
\hline Clorada & 8 & 9,3 & 0,6 \\
\hline Sin tratamiento & 33 & 38,4 & - \\
\hline \multicolumn{4}{|l|}{ Cuarto dormitorio } \\
\hline Condición de hacinamiento & 16 & 18,6 & $2,8^{*}$ \\
\hline Compartido con otra persona & 67 & 78,0 & 0,5 \\
\hline Individual & 3 & 3,5 & - \\
\hline \multicolumn{4}{|l|}{ Presencia de vectores mecánicos } \\
\hline Sí & 57 & 66,3 & 0,3 \\
\hline No & 29 & 33,7 & - \\
\hline \multicolumn{4}{|l|}{ Manejo de excretas } \\
\hline Taza sanitaria & 81 & 94,2 & 0,6 \\
\hline Pozo séptico & 3 & 3,5 & - \\
\hline Fecalismo aire libre & 2 & 2,3 & - \\
\hline \multicolumn{4}{|l|}{ Presencia de mascotas en el hogar } \\
\hline Sí & 38 & 44,2 & 0,0 \\
\hline No & 48 & 55,8 & - \\
\hline \multicolumn{4}{|l|}{ Hábito de lavado de manos } \\
\hline Antes del consumo de alimentos & 57 & 66,3 & 0,3 \\
\hline Después del uso sanitario & 61 & 71,0 & 0,3 \\
\hline $\begin{array}{l}\text { Después del contacto con } \\
\text { mascotas }\end{array}$ & 47 & 54,7 & 0,8 \\
\hline No acostumbra & 7 & 8,1 & - \\
\hline \multicolumn{4}{|c|}{ Lavado de frutas y verduras para consumo } \\
\hline Sí & 63 & 73,3 & - \\
\hline No & 23 & 26,7 & - \\
\hline \multicolumn{4}{|l|}{ Hábito de caminar descalzo } \\
\hline Sí & 49 & 57,0 & 0,3 \\
\hline No & 37 & 43,0 & - \\
\hline \multicolumn{4}{|l|}{ Hábito de jugar con tierra } \\
\hline Sí & 41 & 27,7 & 0,6 \\
\hline No & 45 & 52,3 & - \\
\hline \multicolumn{4}{|l|}{ Hábito de onicofagia } \\
\hline Sí & 6 & 7,0 & 0,2 \\
\hline No & 80 & 93,0 & - \\
\hline
\end{tabular}




\section{Discusión}

En Colombia se han realizado estudios de parasitismo intestinal, que han incluido la enterobiasis y han diferido en el reporte de los valores porcentuales. Así, obedeciendo a esta discrepancia y al tipo de técnica diagnóstica empleada en este, para la evaluación de la parasitosis en la presente investigación se tiene en cuenta la recomendación de la oms en cuanto al método de referencia.

\section{Técnica de diagnóstico}

La técnica de referencia para el diagnóstico de la oxiuriasis, según la oms y la Organización Panamericana de Salud (ops), es el método de Graham, aunque se han evaluado otros de tipo comercial como la cinta adherente Pin Tape-PT, que se coloca en el área anal luego de conciliación del sueño y se retira al día siguiente, con periodo de aplicación entre cinco y seis horas; posteriormente, se almacena en un sobre hasta el análisis microscópico. El uso del método Pin Tape resulta poco práctico porque debe permanecer durante toda la noche, en tanto que la técnica de Graham se obtiene en el momento de la aplicación, lo que la hace menos dispendiosa. En algunos estudios se han evaluado ambos métodos; el de Graham además de su practicidad presentó mayor sensibilidad al detectar un número más elevado de huevos por lámina $(23,25)$.

\section{Evaluación parasitológica}

El estudio más reciente que haya reportado valores de prevalencia para la oxiuriasis en Colombia es la ENPI, la cual realizó el diagnóstico por copropasitología e informó un $1 \%$ de prevalencia general para el país (14). En cuanto a los estudios realizados localmente, los reportes obtenidos son diversos: $10,5 \%$ para población infantil residente en un sector de Ciudad Bolívar, Bogotá, en 1993 (13); 8,6\% en Bucaramanga, Santander, en población infantil de padres desplazados en 2000 (26); $2,5 \%$ en zona rural para la vereda La Virgen del municipio de Quipil, Cundinamarca, en el periodo comprendido entre 2001 y 2003 (12), y 32,9\% en un jardín infantil de Popayán, Cauca, en 2007 (1).

La prevalencia en esta investigación fue de $22,1 \%(19 / 86)$, un valor intermedio a lo registrado a nivel nacional, que se consiguió con una sola muestra utilizando la técnica de Graham. Es importante resaltar la importancia de hacer un seriado, como el que se llevó a cabo en la investigación en Popayán; allí se destaca que con la cuarta muestra se encontraron más infantes positivos. Esta situación es similar a la que se presentó con el estudio realizado en Noruega, la cual incrementó hasta el $90 \%$ la positividad (27).

Los valores dados por otros países latinoamericanos denotan lo prevalente de la oxiuriasis en la población infantil; así, se destacan ciudades como Barquisimeto, en Venezuela, con el $17,7 \%$ en 191 preescolares con edades de 2 a 6 años. En este lugar se identificaron factores epidemiológicos asociados a la parasitosis, como disposición inadecuada de excretas en un 57,1 \% y ausencia de tratamiento antiparasitario en los últimos seis meses previos a la realización del estudio en el 47,3\% de los casos (28). También está Ribeirao Preto Sao Paulo, en Brasil, con 5,6\% y 7,3\% en 962 niños evaluados entre 3 y 12 años de edad (29); en Perú las prevalencias variaron de un área a otra, desde $27 \%$ hasta $79,5 \%$, distribuyéndose de la siguiente manera: guarderías de Arequipa con 34,4\% en niños de 1 a 6 años; Tacna, en escolares de 6 a 16 años, presenta el 32,98\%; en el distrito limeño del Rímac, en un rango etario similar, hay un $32,2 \%$, mientras que en Chiclayo se informó del 27,89\% en escolares en general (25). Una condición similar se observó en Argentina, en las provincias estudiadas donde los valores porcentuales fueron elevados; en este país están afectados principalmente niños y niñas escolares que aún no incorporaron hábitos higiénicos adecuados, con práctica frecuente de onicofagia y deficiente lavado de uñas y manos, hacinamiento en el lugar de residencia, compartir camas y ropa, entre otros, constituyeron factores predisponentes para la parasitosis (30).

Valores análogos se han informado para otros países que reseñan prevalencias elevadas y las atribuyen a factores que se relacionan directamente con el nivel de educación, las condiciones de vida, el hacinamiento y los hábitos de higiene inadecuados. Por ejemplo, en Chile hay prevalencia en un $33 \%$ de adultos; en Venezuela, en el $57,7 \%$ en población en general; en Belize, $58,5 \%$ en niños 
de 0,0 a 4 años, y en Cuba, $35 \%$ en círculos infantiles. En países asiáticos como Irán, 17,2\%, Korea $10,7 \%$, Turquía 5,1\% y Taiwán 0,62\%; este último reportó mayor tasa de infección para la población infantil masculina que para la femenina. Es de anotar que algunos estudios resaltan la relación directa encontrada entre la densidad poblacional y elevadas tasas de enterobiasis $(17,18,31,32)$.

\section{Variables evaluadas}

Las variables relacionadas con la oxiuriasis documentadas en la literatura evidencian asociación con viviendas deficientes, manejo inadecuado de excretas, consumo de agua no tratada, ausencia de tratamiento antiparasitario en los últimos seis meses, desconocimiento de los padres de la parasitosis, el hábito en los menores de "chupar dedo", corte de uñas poco frecuente y fómites, entre otras. En la presente investigación las variables que mostraron valores de $\mathrm{p} \leq 0,05$ y OR con significancia estadística para la adquisición de la parasitosis fueron: poseer computador como elemento fómite y hacinamiento en el cuarto dormitorio del menor $(25,33)$.

Los controles médicos mensuales, trimestrales y vinculación al Sisbén fueron factores protectores que concuerdan con otras investigaciones que destacan el suministro de tratamientos antiparasitarios, intervención gubernamental con dotación de sistemas sanitarios e implementación de saneamiento ambiental; sumado a esto, las actividades de educación en las comunidades evaluadas lograron la reducción del parasitismo intestinal en general en la población estudiada $(19,26,34)$.

La presencia de vectores mecánicos en el lugar de residencia o cerca de esta fue una variable con significancia, pero con poca asociación, a pesar de estar ampliamente relacionada con la transmisión de parasitismo intestinal. En la presente investigación no se evidenció una relación directa entre estos y la infección por oxiuros, lo puede obedecer a que en la mayoría de los casos la infección puede darse anal-oral, a través de ropa interior o de cama, o migración retrógrada de estadios larvales del área perianal al tracto intestinal (19).

Gracias a otras variables evaluadas como fecalismo al aire libre, el uso de antiparasitarios domésticos, la toma de medicamentos no recetados y el consumo de agua no clorada se halló asociación con parasitismo intestinal para otras entidades evaluadas en un estudio paralelo, pero no para oxiuros. Es de suponer que esto obedece a que dicha parasitosis no es una geohelmintiasis y además es poco frecuente el hallazgo de huevos en heces; también, está más asociada a fómites (ropa interior, de cama) y alimentos contaminados directamente por personas parasitadas. Estudios realizados en Suráfrica demostraron la presencia de huevos de E. vermicularis en las manos de los menores, y argumentan que estas cumplen el papel de fómite en la adquisición de la helmintiasis. Aunque no se halló asociación estadística con sintomatología alguna y los menores presentaron buena condición nutricional en el momento del estudio, algunos refirieron presentar ocasionalmente prurito nasal y anal $(35,36,37)$.

\section{Conclusión}

La prevalencia para oxiuriasis fue de 22,1\% (19/86), $27,3 \%(12 / 44)$ en Chaguaní y 16,7\% (7/42) en Cajicá. Lo resultados pueden indicar que la prevalencia de oxiuriasis es más dependiente de condiciones higiénico sanitarias de índole personal que medioambientales, factor de riesgo que es ampliamente manifestado por varios autores en estudios realizados en diversas comunidades que habitan en lugares que difieren en estos parámetros. Entre las variables evaluadas, se halló significancia estadística para la adquisición de la parasitosis la condición de hacinamiento en el cuarto del menor y poseer computador como un elemento fómite, por el contacto que pueden tener los menores con este aparato para actividades académicas y recreativas. Aunque varios autores reportan en sus estudios mayor prevalencia de la oxiuriasis en el género masculino, para el caso de la presente investigación fue el femenino el de mayor infección.

\section{Agradecimientos}

A la comunidad de Chaguaní, a las autoridades gubernamentales y de salud municipal, a las directivas de los jardines infantiles, Sandra Ceballos (CAFAM) y Carina Santacoloma, de Cajicá, y a la comunidad participante, asimismo, a los estudiantes de la Facultad de Medicina de la Universidad 
Militar Nueva Granada y al personal del laboratorio del programa profesional de Biología de la Universidad Incca de Colombia.

\section{Financiación}

La presente investigación fue financiada con recursos propios del Grupo de Investigación en Parasitología y Microbiología Tropical (GIPAMT) de la Universidad Incca de Colombia y por el Semillero de Investigación en Enfermedades Infecciosas de la Facultad de Medicina de la Universidad Militar Nueva Granada.

\section{Conflicto de interés}

Los autores declaramos no presentar conflicto de intereses en la presente investigación. Todas las muestras biológicas fueron tratadas acorde con la normatividad bioética y los resultados de la población participante fueron informados de manera personal y confidencial a los padres o tutores de los menores.

\section{Referencias}

[1] Martínez Andrade S, Acosta A, Bogorje G, Vásquez LR, González FE, Alvarado BE. Prevalencia de Enterobius vermicularis en niños de 3 meses a 6 años de un hogar infantil en Popayán [Internet. Biomed Rev del Inst Nac Salud. 2007;23. Disponible en: https://www.researchgate.net/publication/237763015

[2] Acosta M, Cazorla-Perfetti DJ, Garvett M. Enterobiasis en escolares de una población rural del Estado Falcón, Venezuela y su relación con el nivel socio-económico [Internet]. Invest Clin. 2002;43(3):173-181. Disponible en: http://ve.scielo.org/scielo.php?script=sci_arttext\&pid=S0535-51332002000300004

[3] Cazorla-Perfetti D. Aspectos relevantes de la enterobiosis humana. Revisión crítica [Internet]. 2014;26(3):221242. Disponible en: https:/www.redalyc.org/ pdf/4277/427739473002.pdf

[4] Iannacone J, Benites MJ, Chirinos L. Prevalence of intestinal parasitic infection in primary schoolchildren of Santiago de Surco, Lima, Peru [Internet]. Parasitol. Latinoam. 2006;61(1-2):54-62. Disponible en: https:// www.researchgate.net/publication/289803002_Prevalence_of_intestinal_parasitic_infection_in_primary_ schoolchildren_of_Santiago_de_Surco_Lima_Peru

[5] Ayala M de la M, Monroy F, Gómez E M. Enterobiasis vaginal. Comunicación de un caso [Internet]. Ginecol
Obstet Mex. 2009;77(10):491-3. Disponible en: http:// www.medigraphic.com/pdfs/ginobsmex/gom-2009/ gom0910g.pdf

[6] Tashima NT, Simões, MJ. Parasitas intestinais; prevalência e correlação com a idade e com os sintomas apresentados de uma população infantil de Presidente Prudente-SP [Internet]. Rev. Bras Anal Clin.2005;37(1):35-39. Disponible en: http://www.scielo.br/scielo.php?script $=$ sci_nlinks\&ref=000114\&pi$\mathrm{d}=$ S0037-8682200800030000800024\&lng=pt

[7] Tapia O, Muñoz C. Oxiuriasis apendicular: estudio de prevalencia y descripción clínico-morfológica [Internet]. Rev Chil Cir. 2011;63(6):599-603. Disponible en: http://dx.doi.org/10.4067/S0718-40262011000600009

[8] Dos Santos VM, De Oliveira Silva MB, Monteiro Godoi J, De Lima MA. Granulomatous nodule with Enterobius vermicularis in epiploon simulating metastasis of ovarian cáncer [Internet]. Rev Soc Bras Med Trop. 2002;35(2):91-93. Disponible en: http://dx.doi. org/10.1590/S0037-86822002000200010

[9] Fernandes da Silva D, Da Silva RJ, Da Silva MG, Cervantes Sartorelli A, Katsunori Takegawa B, Marchesan Rodrigues M A. Infecções parasitárias do apêndice cecal e suas relações com apendicite aguda [Internet]. Arq Gastroenterol. 2008;45(2):166-168. Disponible en: http://dx.doi.org/10.1590/S0004-28032008000200015

[10] Babady NE Awender E, Geller R, Miller T, Scheetz G. Arguello $\mathrm{H}$, et al. Enterobius vermicularis in a 14-Year-Old Girl's Eye [Internet]. J Clin Microbiol. 2011 dic.;49(12):4369-4370. Disponible en: http://dx. doi:10.1128/JCM.05475-11

[11] Boga JA, Rojo S, Fernández J, Rodríguez M, Iglesias C, Martínez-Camblor $\mathrm{P}$, et al. Is the treatment of Enterobius vermicularis co-infection necessary to eradicate Dientamoeba fragilis infection? [Internet]. Int J Infec Dis. 2016;49:59-61. Disponible en: https://www.ncbi. nlm.nih.gov/pubmed/27263118

[12] Knudson A, Lemos E, Ariza Y, Salazar M, Chaves M del P, Reyes P, et al. Frecuencia de E. vermicularis en una población Escolar Rural de Quipile, Colombia. 2001 [Internet]. Rev Salud Púb. 2003;5(1):1-10. Disponible en: http://www.scielo.org.co/scielo.php?script=sci_arttext\&pid=S0124-00642003000100007

[13] Rodríguez C, Mejía L. Prevalencia de Enterobius vermicularis en el centro de atención del Barrio la Victoria. Bogotá. Biomédica. 1993;13:88-9.

[14] Ministerio de Salud y Protección Social. Encuesta Nacional de parasitismo intestinal en población escolar Colombia, 2012-2014 [Internet]. Medellín: Análisis en profundidad. Universidad de Antioquia, Instituto Nacional de Salud; 2015. 174 p. Disponible en: https:// 
www.minsalud.gov.co/sites/rid/Lists/BibliotecaDigital/RIDE/VS/PP/ET/encuesta-nacional-de-parasitismo-2012-2014.pdf

[15] Municipios de Colombia. El municipio de Cajicá [Internet]. Disponible en: https://www.municipio.com. co/municipio-cajica.html

[16] Kang IS, Kim DH, An HG, Son HM, Cho MK, Park $\mathrm{MK}$, et al. Impact of health education on the prevalence of enterobiasis in Korean preschool students [Internet]. Acta Trop. 2012;122(1):59-63. Disponible en: http://www.sciencedirect.com/science/article/pii/ S0001706X11003433

[17] Pérez Cueto M del C, Sánchez Álvarez M de L, Cueto Montoya GA, Mayor Puerta AM, Fernández Cárdenas $\mathrm{N}$, Alegret Rodríguez M. Intervención educativa y parasitismo intestinal en niños de la enseñanza primaria [Internet]. Rev Cubana Med Gen Integr. 2007;23(2). Disponible en: http://scielo.sld.cu/scielo.php?script=sci_arttext\&pid=S0864-21252007000200010

[18] Mugeb OA., Baykan Z., Artan C. Enterobiasis among preschool children: A study from Kayseri, Turkey. [Internet]. Jpn J Infect Dis. 2008 nov.;61:482-3. Disponible en: http://www.ncbi.nlm.nih.gov/pubmed/19050361

[19] Chang TK, Liao CW, Huang YC, Chang CC, Chou CM, Tsay HC, et al., Prevalence of Enterobius vermicularis. Infection among preschool children in kindergartens of Taipei City, Taiwan in 2008 [Internet]. Korean J Parasitol. 2009;47(2):185-7. Disponible en: https://doi.org/10.3347/kjp.2009.47.2.185

[20] Chaguaní-Cundinamarca. Sitio web del municipio Chaguaní en Cundinamarca. Alcaldia de Chaguaní [Internet]. 2015. Disponible en: http://www.chaguani-cundinamarca.gov.co/

[21] Saldaña-Angel LH. Plan de desarrollo municipal 20122015 [Internet]. Chaguaní; 2015. Disponible en: http:// cdim.esap.edu.co/BancoMedios/Imagenes/chachaguanicundinamarcapd2012-2015.pdf

[22] Alzate P. Aprobada la nueva Ley de Protección al Menor [Internet]. 2015 jul. 24. Disponible en: http://www. am-abogados.com/blog/aprobada-la-nueva-ley-deproteccion-al-menor/5790/

[23] Báez López N, Pereira Boan J, Ruiz Aliende S, Marne Trapero C. Prueba de Graham y enterobiasis; resultados de 11 años [Internet]. Rev Pediatr Aten Primaria. 2013;15(57):e1-e3. Disponible en: http://dx.doi. org/10.4321/S1139-76322013000100005

[24] Centro para el Control y la Prevención de Enfermedades (CDP). Epi Info en español [Internet]. Disponible en: https://www.cdc.gov/epiinfo/support/esp/ es_downloads.html
[25] Beltrán MF, Hara T, Raúl C. Evaluación de los métodos de Graham y Pin Tape en el diagnóstico de Enterobius vermicularis [Internet]. Rev Perú Med Exp Salud Publica.2005;22(1):76-78, Disponible en: http://www. scielo.org.pe/pdf/rins/v22n1/a12v22n1.pdf

[26] Arazona Z, Farfan A, Afanador A, Amorocho D, Castillo M, Fernández M. Parásitos intestinales en niños desplazados en Santander. Rev Salud UIS. 2000;32:89-92.

[27] Bøås H, Tapia G, Sødahl JA, Rasmussen T, Rønningen KS. Enterobius vermicularis and risk factors in healthy norwegian children [Internet]. Pediatr Infect Dis J. 2012;31(9):927-930. Disponible en: https://doi. org/10.1097/INF.0b013e318258cdb5.

[28] Cervantes J, Otazo G, Rojas M, Vivas F, Yousseph Y, Zechini V. Enteroparasitosis, enterobiasis y factores de riesgo en niños preescolares. Salud, Arte y Cuidado. 2012;5(1):47-54. Disponible en: https://dialnet.unirioja.es/servlet/articulo? codigo $=4117696$

[29] Fonseca REP, Barbosa MCR, Ferreira BR. High prevalence of enteroparasites in children from Ribeirão Preto, São Paulo, Brazil. Rev Bras Enferm [Internet]. 2017;70(3):566-71. Disponible en: http://dx.doi. org/10.1590/0034-7167-2016-0059

[30] Navone GT, Zonta ML, Cociancic P, Garraza M, Gamboa MI, Giambelluca LA, et al. Estudio transversal de las parasitosis intestinales en poblaciones infantiles de Argentina [Internet]. Rev. Panam. Salud Pública. 2017;41:e24. Disponible en: http://www.scielosp.org/ pdf/rpsp/v41/1020-4989-RPSP-41-e24.pdf

[31] Chen KY, Yen CM, Hwang KP, Wang LC. Enterobius vermicularis infection and its risk factors among preschool children in Taipei, Taiwan [Internet]. J. Microbiol. Immunol. Infect. 2017;51(4). Disponible en: https://doi.org/10.1016/j.jmii.2016.12.013

[32] Kim DH, Son HM, Kim JY, Cho MK, Park MK, Kang SY, et al. Parents' knowledge about enterobiasis might be one of the most important risk factors for enterobiasis in children. Korean J Parasitol. 2010;48(2):121-126. Disponible en: https://pdfs.semanticscholar.org/3806/ da38a3a61417912c2df8761534c005077036.pdf

[33] N. Keskin, A. Ay Bektaş. The prevalence of Enterobius vermicularis in primary school which have different socioeconomic level in Ankara [Internet]. Turk Parazitol Derg. 2014;38(3):159-165. Disponible en https:// doi.org/10.5152/tpd.2014.3557

[34] Kang IS, Kim DH, An HG, Son HM, Cho MK,Park $\mathrm{MK}$, et al. Impacto de la educación sobre la salud en la prevalencia de enterobiasis en los estudiantes de preescolar coreanos [Internet]. Acta Trop. 2012;122(1):59-63. Disponible en: https://doi.org/10.1016/j.actatropica.2011.11.017 
[35] Giraldo JC, Chala DM, Vásquez LR, Zamora TÓ, Casas JC. Sintomatología asociada a neurocisticercosis en personas del área urbana y periurbana del municipio de Coyaima del departamento del Tolima y posibles variables asociadas [Internet]. Rev Med 2016;24(2):822: Disponible en: https://doi.org/10.18359/rmed.2626

[36] Ramírez JD, Flórez C, Olivera M, Bernal MC, Giraldo JC. Blastocystis subtyping and its association with intestinal parasites in children from different geographical regions of Colombia [Internet]. PLOS ONE. 2017 febr. 21;12(2): e0172586. Disponible en: https:// doi.org/10.1371/journal.pone.0172586
[37] Cranston I, Potgieter N, Mathebula S, Ensink JH. Transmission of Enterobius vermicularis eggs through hands of school children in rural South Africa [Internet]. Acta Trop. 2015;150:94-6. Disponible en: https:// doi.org/10.1016/j.actatropica.2015.07.001 